\title{
BER Improvement Using Optical Gain Clamped Amplifier for Burst Transmission and Critical Cases Studies
}

\author{
M. Zannin ${ }^{1}$, K. Ennser ${ }^{1}$, S.Taccheo ${ }^{1}$, D. Careglio ${ }^{2}$, J. Solé-Pareta $^{2}$, J. Aracil $^{3}$ \\ 1: Institute of Advanced Telecommunications, Swansea University, Swansea SA2 8PP, UK, e-mail: k.ennser@swansea.ac.uk \\ 2: Universitat Politècnica de Catalunya, Jordi Girona, 1-3, 08034 Barcelona, Catalonia, Spain \\ 3: Universidad de Madrid, Madrid, Spain
}

\begin{abstract}
This paper demonstrates significant BER improvement in burst traffic transmission using optical-gain clamped amplifiers. Critical chaotic cases are identified for peculiar burst sequences and their impact in transmission is assessed.

(C)2008 Optical Society of America

OCIS codes: (060.2320) Fiber optics amplifiers and oscillators; (060.4510) Optical communications.
\end{abstract}

\section{Introduction}

Strong signal power variations due to both signal add/drop or burst transmission are a main concern in amplified transmission systems due to gain saturation of optical amplifiers (OA). In particular this is a key issue for optical burst transmission [1], where potential degradation can be accumulated along the system [2]. Several techniques have been proposed to stabilize the amplifier gain and passive all-optical means is a very promising one [3]. In particular, all-optical gain clamping of Erbium-doped amplifiers in optimized condition have successfully proved to strongly reduce overshoot after add/drop transients [4], and allowed for the first time stable close-loop network operation [5]. Recent work has demonstrated that overshoot induced by burst transmission may also be significantly reduced by optical-gain-clamped (OGC) technique [6]. However, no direct bit-error-rate (BER) improvement has been assessed. In addition, the burst mode transmission may interplay with intrinsic chaotic dynamics of OGC-OA.

This paper reports BER improvement in WDM burst transmission and the BER performance in critical cases when burst duration and burst inter-arrival time interplay with OGC-OA dynamics. In addition, the paper investigates the burst traffic characteristics to evaluate the probability of critical case occurrence. The results demonstrate that OGC-OA brings significant BER improvement for burst traffic and critical case occurrence will not impact the BER performance.

\section{Setup Description}

The data used to simulate the WDM burst transmission was obtained from a pair of Full-Duplex Gigabit Ethernet links, which connects the Catalan R\&D network (around 50 Universities and Research Centers) with the Spanish R\&D RedIris network and to the internet [7,8]. An optical burst switching (OBS) node performs packets aggregation and burst generation [7]. A 14-s long trace with 95k burst is obtained from the testbed and used to build a 1-s long WDM burst stream by taking uncorrelated data from different time slots [7].

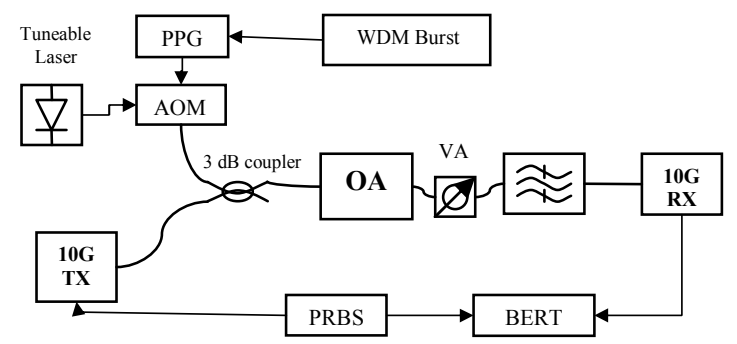

Fig. 1. Experimental setup for the multi-channel burst transmission

The experimental set-up is depicted in Fig.1. The signal obtained from 15 WDM burst traffic channels controls the pulse pattern generator (PPG) driving the acousto-optic modulator (AOM) to modulate a tunable laser source. The maximum power after the AOM entering the commercial erbium-doped fiber amplifier (EDFA) is $-1 \mathrm{dBm}$ and simulates $15 \mathrm{WDM}$ channels at $1550 \mathrm{~nm}$. A continuous wave probe signal simulating the $16^{\text {th }}$ channel is used for BER measurement. This channel is implemented with a pseudo-random binary sequence generator (PRBS) and a 10 Gbit/s transmitter at $1556.5 \mathrm{~nm}$ with $-15 \mathrm{dBm}$ average power. The commercial EDFA used on these experiments is 


\section{OTuH3.pdf}

configured for a 17-dB gain and has noise figure of $6.5 \mathrm{~dB}$ [6]. The amplifier is tested in clamped and unclamped conditions under same gain and input power conditions. To achieve optical feedback, for sake of simplicity and without affecting the test validation, a ring configuration with cavity length of about $7 \mathrm{~m}$ is used. A variable attenuator (VA) simulates transmission losses and a tunable filter enables probe channel reception.

\section{Amplifier dynamic characterization and critical case investigation}

Before performing transmission experiments, the dynamic behavior of the amplifier is evaluated for specific burst sequences. As demonstrated in [8], the interplay of burst duration/inter-arrival time with OGC-OA relaxation oscillation frequency (i.e. with intrinsic frequency the OGC-OA relaxing to steady state after perturbation) may generate chaotic behavior, as shown in Fig. 2. The OGC-OA is tested using the $-1 \mathrm{dBm}$ signal on/off modulated.

First measurements evaluate the add/drop relaxation oscillation frequencies (ROF) [8], to be 13.5 and $23.5 \mathrm{kHz}$, respectively. Subsequently, the frequency $f$, which represents the simultaneous on/off of all the channels, is swept from $1 \mathrm{~Hz}$ to $200 \mathrm{kHz}, 50 \%$ duty cycle, and backward. Fig. 2 shows the overshoot observed as a function of the burst inter-arrival time $T(T=1 / 2 f)$. During the forward sweep (with respect to time), when the burst duration and inter-arrival time are increased, it is possible to note a peak for burst of $21.3 \mu \mathrm{s}$, followed by a peak at $37 \mu \mathrm{s}$ related to drop and add frequency, respectively. This is due to interplay between burst sequence and intrinsic OGC-OA gain recovery dynamic [9]. It may be inferred that sequences of bursts with duration and inter-arrival times between $10 \mu \mathrm{s}$ and $80 \mu$ s may generate chaotic behavior with potential BER transmission performance penalty.
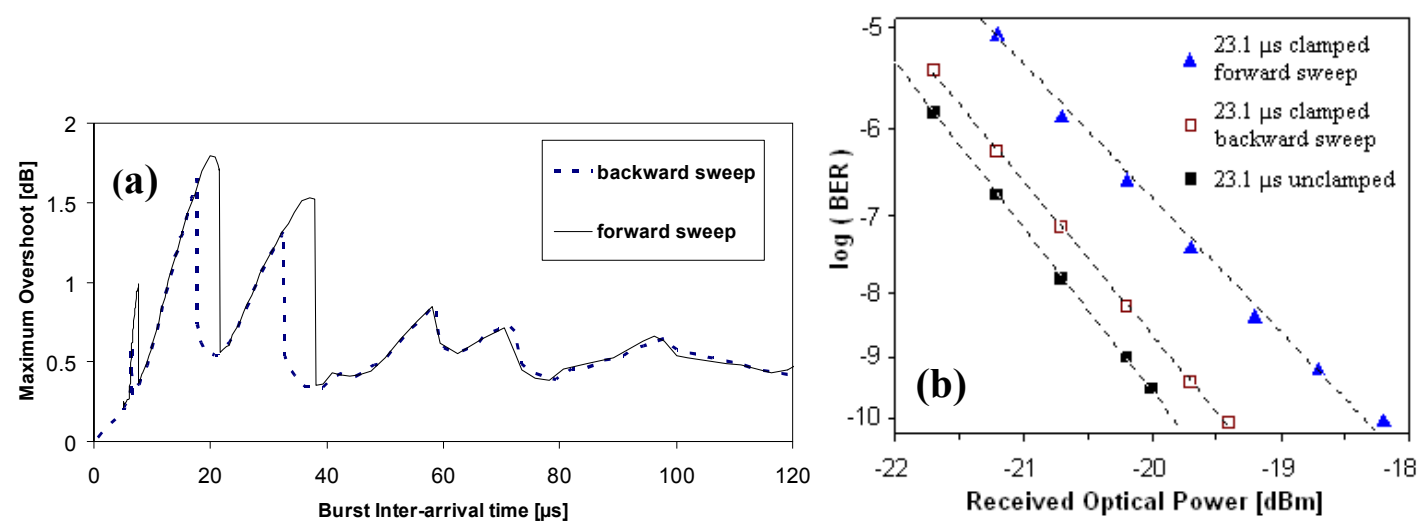

Fig. 2. (a) Maximum power overshoot for on/off modulation. Time scale refers to burst duration with $50 \%$ duty cycle: b) BER for on/off with burst of 23.1 microseconds (50\% duty cycle) for clamped (forward and backward sweep) and unclamped condition.

BER measurements are performed in order to assess this issue, as shown in Fig. 2b. A BER penalty quite significant is observed for the forward sweep while very similar behavior for unclamped and backward sweep is also noted. For this worst case scenario with 15 simultaneous bursts with same duration and inter-arrival times, it is possible to observe that for the highest overshoot peak, the clamped amplifier introduces a transmission penalty of around $1.7 \mathrm{~dB}$ at $\mathrm{BER}=10^{-10}$. The good performance of unclamped $\mathrm{OA}$ is due to the fact that power variation due to short bursts is averaged by the ms-time scale dynamic of unclamped OA.

Next step is, therefore, the assessment of burst occurrence with duration and/or inter-arrival time between $20 \mu \mathrm{s}$ and $80 \mu$ s. Fig. 3 shows the calculated statistic of the burst traffic data obtained from the OBS network and it is observed that most bursts and most of inter-arrival times fall in the potentially dangerous region $20 \mu \mathrm{s}-80 \mu \mathrm{s}$.
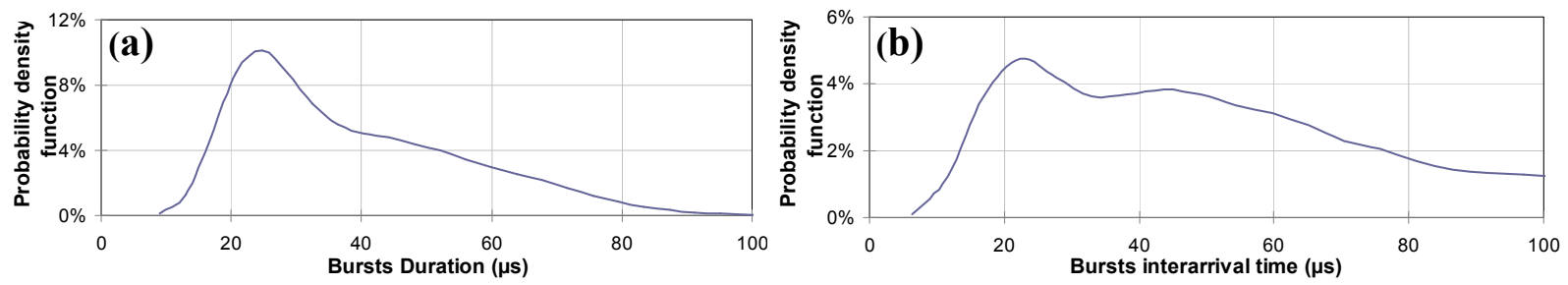

Fig. 3. Occurrence statistic of burst durations (a) and inter-arrival times (b).

\section{Burst transmission experiment}

The BER measurement is performed using the burst traffic data (see Fig. 4). The OGC-OA performs with no extra penalty with respect to the back-to-back BER measurement with traffic data, while unclamped OA exhibits a 


\section{OTuH3.pdf}

penalty of $1 \mathrm{~dB}$ for BER $=10^{-10}$. Figure 4 also shows BER values when the channels are simultaneously switched on/off at a $1 \mathrm{kHz}$ repetition rate. In this case, the OGC-OA again shows no penalty while the unclamped OA shows a penalty of over $3 \mathrm{~dB}$ for $\mathrm{BER}=10^{-10}$. This last result is very indicative of the possibility of implementing synchronized WDM OBS transmission (all burst channels are aligned) using OGC-OA. The OGC-OA excellent performance with traffic data can be explained with the following considerations. Firstly, while it is true that burst falls in the dangerous region our simulations have shown that to initiate the chaotic behavior, 4 to 5 bursts in sequence are needed. The probability of having such a sequence is very low [10]. Furthermore, the chaotic behavior induced penalty grows nonlinearly with the amount of power switched on/off. In case of non aligned burst channels, as in burst traffic data, the power excursion is limited. In fact, the probability of having all bursts synchronized to generate add/drop like transient is almost zero. However, the chaotic behavior observed in the worst case scenario may be avoided by shifting the resonating frequency out of burst statistics. This can be done by adjusting the OGCOA operating condition and/or the resonating cavity length.
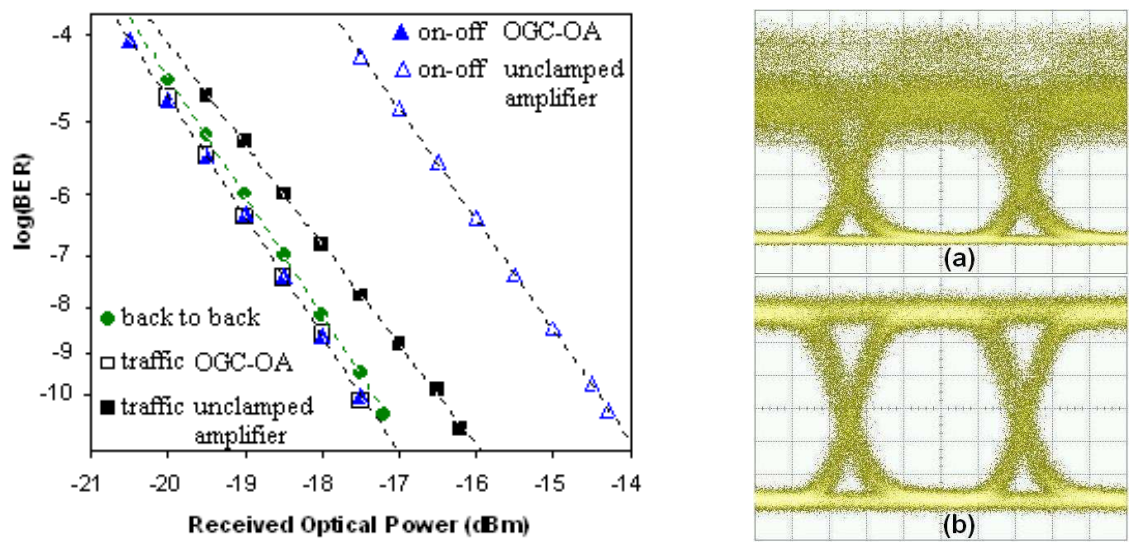

Fig. 4. Left: Bit Error Rate curves for the probe channel with burst traffic data and for $1 \mathrm{kHz}$ simultaneous drop and addition of all channels. Right: Eye-diagrams for burst traffic scenario using (a) unclamped OA and (b) OGC-OA.

\section{Conclusions}

This paper demonstrates that OGC-OA has no penalty with respect to back-to-back burst transmission and also shows a $1 \mathrm{~dB}$ improvement at BER of $10^{-10}$ with respect to unclamped OA. In addition, no penalty is shown for channels add/drop while $3 \mathrm{~dB}$ penalty at BER of $10^{-10}$ is measured for unclamped OA. Furthermore, the results show that while chaotic behavior may generate BER penalty in burst traffic condition, there is almost negligible probability of initiating such a chaotic behavior. The conclusion is that OGC-OA offers BER performance benefits versus unclamped OA for burst transmissions.

Acknowledgments: The work was carried out with the support of the BONE-project ("Building the Future Optical Network in Europe"), a Network of Excellence funded by the European Commission through the 7th ICT-Framework Programme and by the Welsh Assembly Government.

\section{References}

[1] Yu-Li Hsueh, J. Kim, Ching-Fong Su, R. Rabbat, T. Hamada, C. Tian and L.G. Kazovsky, "Traffic grooming on WDM rings using optical burst transport," J. Lightwave Technol., vol. 24, pp. 44-53, 2006.

[2] A. Lieu, C. Tian, T. Naito, "Transmission and interactions of WDM burst signals in cascaded EDFAs," OFC'06, USA, paper OtuD5.

[3] Zirngibl, M., "Gain control in erbium-doped fibre amplifiers by an all-optical feedback loop", Electron. Lett., Vol. 27, , pp. 560-561, 1991

[4] K. Ennser, G. Della Valle, M. Ibsen, J. Schmulovich and S. Taccheo, "Erbium-doped waveguide amplifier for reconfigurable WDM metro networks", IEEE Phot. Tech. Lett., vol. 17, pp. 1468-1460, 2005.

[5] K. Ennser, S. Taccheo, T. Rogowski, and J. Shmulovich, "Optical Stabilization of Waveguide Amplifiers for WDM Ring Network With Recirculating Optical Power," J. Lightwave Technol. 25, 1670-1675 (2007)

[6] M. Zannin, S. Taccheo, K. Ennser, D. Careglio, and J. Solé-Pareta, "Effective Amplification of Real WDM Burst Traffic Using Optical Gain Clamping," in Prof. CLEO 2008 paper CThAA6.

[7] P. Barlet, J. Sole Pareta, J. Barrantes, E. Codina, J. Domingo, "SMARTxAC: A Passive Monitoring and Analysis System for High-Speed Networks", in Proc. Tyrrean Networking Conference, Italy.

[8] S. Taccheo, A. Festa G. Della Valle and K. Ennser "Gain-stabilized Erbium-Doped Waveguide Amplifier for Burst Transmission", IEEE Photon. Technol. Lett., 19, 97-99 (2007)

[9] G. Della Valle, A. Festa, S. Taccheo, K. Ennser and J. Aracil " Nonlinear dynamics induced by burst amplification in optically gainstabilized erbium-doped amplifiers", Optics Letters, 32, 903-905, 2007

[10] S. Taccheo, G. Della Valle, A. Festa and K. Ennser, "Amplification of Optical Bursts in Gain-Stabilized Erbium-Doped Optical Amplifier", OFC2007, USA, paper OMN3 